Document downloaded from:

http://hdl.handle.net/10251/178899

This paper must be cited as:

Jiménez-Gambín, S.; Jimenez, N.; Benlloch Baviera, JM.; Camarena Femenia, F.; Pouliopoulos, AN.; Konofagou, EE. (2020). First in-vivo Demonstration of Bilateral BloodBrain Barrier Opening Using Acoustic Holograms in Mice. IEEE. 1-4. https://doi.org/10.1109/IUS46767.2020.9251487

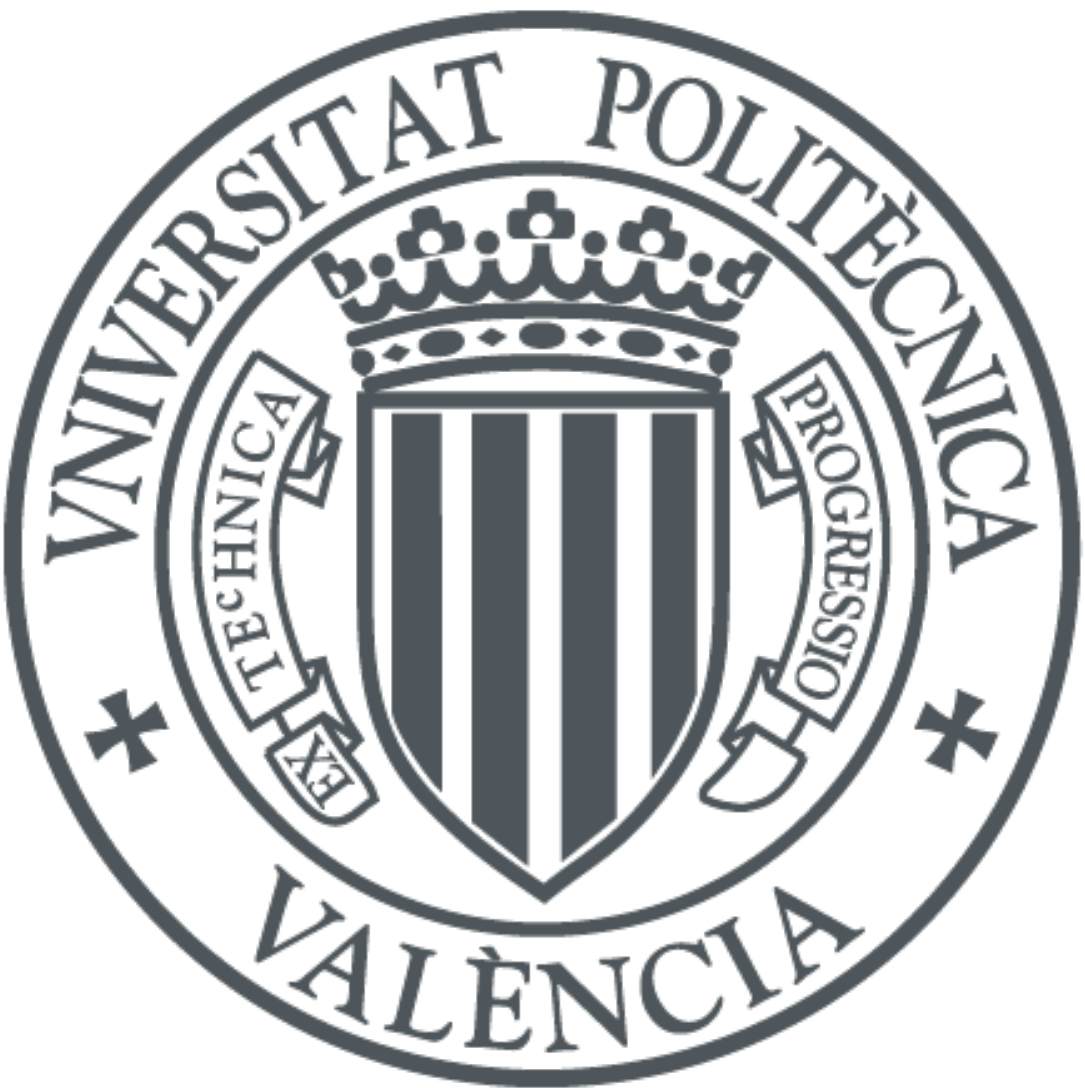

The final publication is available at

https://doi.org/10.1109/IUS46767.2020.9251487

Copyright IEEE

Additional Information 


\section{First in-vivo Demonstration of Bilateral Blood-Brain Barrier Opening Using Acoustic Holograms in Mice}

\author{
Sergio Jiménez-Gambín*, Noé Jiménez, \\ José M. Benlloch and Francisco Camarena \\ Instituto de Instrumentación para Imagen Molecular \\ Universitat Politècnica de València - CSIC, \\ Valencia, Spain \\ *serjigam@upv.es
}

\author{
Antonios N. Pouliopoulos and Elisa E. Konofagou \\ Department of Biomedical Engineering \\ Columbia University, \\ New York City, USA
}

\begin{abstract}
Focused ultrasound (FUS) with microbubbles allows for non-invasive targeted drug delivery into the central nervous system (CNS) by temporally and locally disrupting the bloodbrain barrier (BBB). However, current FUS technologies are not able to simultaneously target several brain structures. In this work, we open the BBB in two regions in a murine brain using a single-element transducer with a coupled 3D-printed holographic lens, which is designed to simultaneously create two symmetric foci in anesthetized mice in vivo. The proposed approach shows many advantages: (1) simple and low-cost; (2) correction of aberrations due to skull and water cone; and (3) multiple BBB opening (BBBO) locations with only one sonication, becoming a time- and cost-effective therapeutic system for neurological diseases. For the in-vivo experiment, contrast-enhanced, T1weighted MRI scan was conducted following BBBO, showing gadolinium extravasation at two symmetric focal spots. The two BBBO regions were separated by $3.0 \pm 0.7 \mathrm{~mm} \quad(\mathrm{n}=5$ mice) compared to $5.3 \mathrm{~mm}$ in full-wave simulations. This work shows the capability of bifocal ultrasound generation in separate animals using a unique $\mu$ CT scan. A bilateral BBBO was achieved with a single sonication using a holographic lens in mice, thus improving the efficiency and defining a new approach for several neurodegenerative diseases targeting symmetric brain structures, e.g. hippocampus, putamen or caudate. This study demonstrates the feasibility of hologram-assisted BBBO for targeted drug delivery in the CNS in symmetric regions in separate hemispheres.
\end{abstract}

Index Terms-acoustic hologram, blood-brain barrier opening, focused ultrasound

\section{BACKGROUND}

The low perfusion of therapeutic drugs into the CNS due to the reduced permeability of the BBB is a limiting factor for the treatment of most brain diseases. However, microbubbleenhanced FUS is a method to produce localized, transient, non-invasive and safe BBBO in rodents [1], [2], non-human primates [3] and humans [4] showing high feasibility for these brain treatments.

Single-element FUS or phased-array systems successfully disrupt the BBB but do not allow for simultaneous multifocal targeting. On the one hand, single-element systems for small animals need a mechanical movement of the transducer for the multi-focal targeting [5], [6]. Several microbubble injections are required since they are rapidly cleared from the bloodstream, becoming a time-inefficient approach. The same limitation is found using neuronavigation systems in non-human primates [3], since the mechanical movement of the transducer is a time-limiting factor. On the other hand, phased-array systems for clinical human trials [4] electronically control the amplitude and phase of each piezoelectric element to steer the focus while the transducer remains in a fixed position. However, these systems are limited by the steering angle, their high-cost and the requirement for MRI guidance.

To overcome these limitations, holographic acoustic lenses have recently shown the generation of simultaneous multifocal targets, acoustic fields matching the focal volume with the geometry of CNS structures, self-bending beams or vortex beams [7]-[10]. Acoustic holography is the alternative to avoid the time-inefficient movement of the FUS transducer and it is a low-cost and simple technology. Furthermore, these lenses are 3D-printable and MRI-compatible, needing just one MRIscan to identify the correct alignment. Moreover, since these lenses correct the skull aberrations, a unique sonication and injection of microbubbles is enough for the entire treatment.

State-of-the-art acoustic holographic lensing has shown success in water tanks, through human-skull phantoms and invitro human-skulls, but not in-vivo. In this study, we demonstrate the feasibility, capability and efficiency of acoustic holography using a FUS single-element transducer for the first time in-vivo, showing a bilateral multi-focal BBBO in mice.

\section{Materials AND Methods}

The proposed method consists of: (1) extracting the geometry and acoustic properties of the skull from X-ray $\mu \mathrm{CT}$ images and identifying the target structure using MRI scans; (2) designing and 3D-printing the plastic cone for the transducerhead water coupling; (3) obtaining the holographic wavefront by simulation of the back-propagation and phase-conjugation analysis; (4) designing the lens from the holographic phase and stereo-lithographic 3D-printing; (5) injecting the microbubbles during sonication; (6) assessing the BBBO using MRI.

\section{A. Numerical simulation}

A pseudo-spectral simulation method with $k$-space dispersion correction was used to numerically integrate the linearized 
constitutive relations of acoustics [11], including a tissue absorption model that follows a power law on frequency. This simulation method is selected as it provides low numerical dispersion as compared with finite-differences methods [12]. We use a numerical grid with a spatial step of $\Delta x=\Delta y=$ $\Delta z=176 \mu \mathrm{m}$, which corresponds to a spatial sampling of 6 grid points per wavelength in water for a frequency of 1.68 $\mathrm{MHz}$, leading to simulations of more than 300 million grid points, and with a numerical temporal step of $\Delta t=13.7 \mathrm{~ns}$, leading to a Courant-Friedrichs-Lewy number of $C F L=0.12$ $\left(C F L=c_{\max } \Delta t / \Delta x\right.$, where $c_{\max }$ is the maximum sound speed in skull bone).

\section{B. CT-scan of the skull of the mouse}

We used a $\mu$ CT-scan with an isotropic spatial resolution of $80 \mu \mathrm{m}$ (under-sampled to $176 \mu \mathrm{m}$ isotropic for the simulation). The heterogeneous density and sound speed were calculated from Hounsfield units using the linear-piecewise polynomials proposed in [13], [14]. The absorption was assumed to be homogeneous [15] and set to $28.3 \mathrm{~dB} / \mathrm{cm}$ at $1.68 \mathrm{MHz}$ [16]. The brain volume was segmented from the cavity in the $\mu \mathrm{CT}$ scan using the ITK-SNAP software [17], and its acoustical properties are $\rho_{\text {brain }}=1000 \mathrm{~kg} / \mathrm{m}^{3}, c_{\text {brain }}=1600 \mathrm{~m} / \mathrm{s}$ and absorption of $0.7 \mathrm{~dB} / \mathrm{cm}$ at $1.68 \mathrm{MHz}$ [1].

\section{Lens design and manufacturing}

The lens was designed from the recorded acoustic complex wavefront at the holographic surface [8] generated by two virtual omni-directional pressure sources located at the targets in each hemisphere of the brain. Unlike the flat-surface singleelement transducer used in the original holographic method [8], in this work we used a focused transducer, so the lens should compensate the parabolic focusing, in addition to the aberrations of the central hole used for the diagnostic transducer and the plastic water-filled coupling cone.

The holographic information to design the lens was calculated as

$$
H_{\mathrm{lens}}(x, y)=H_{T}^{*}(x, y) \cdot H_{F}^{*}(x, y),
$$

where $H_{T}^{*}(x, y)$ is the complex conjugate of the recorded wavefront at the holographic surface generated by the backpropagation of the virtual sources; $H_{F}^{*}(x, y)$ is the complex conjugate of the recorded wavefront at the holographic surface generated by the FUS transducer; and $x$ and $y$ are Cartesian coordinates.

Finally, to calculate the height of each pixel [8], we simplified the design due to the simpler bi-focal geometry by ignoring the magnitude of $H_{\text {lens }}(x, y)$ and using only the phase. Both the lenses and plastic cone manufactured in this work were 3D-printed using ClearResin material and stereolithography techniques (Form2, Formlabs). The sound speed and density were experimentally estimated as $c_{L}=2580$ $\mathrm{m} / \mathrm{s}$ and $\rho_{L}=1171 \mathrm{~kg} / \mathrm{m}^{3}$, respectively, and the absorption was set to $\alpha=4.6 \mathrm{~dB} / \mathrm{cm}$ at $1.68 \mathrm{MHz}$, matching the values reported in the existing literature [7], [8].

\section{BBB opening procedure}

The single-element FUS transducer used was the Sonic Concepts H-204 (1.68 MHz, OD $=84.2 \mathrm{~mm}, I D=43.6$ $\mathrm{mm}, F=61.65 \mathrm{~mm}$ ) mounted confocally with a diagnostic transducer (central frequency of $10 \mathrm{MHz}$ ) to image a metallic grid placed on top of the skull lamboid suture. This system was attached to a computer-controlled, 3D positioning system (Velmex Inc., Lachine, QC, CAN). The FUS transducer was driven by a function generator (Agilent, Palo Alto, CA, USA) and a 50-dB power amplifier (ENI Inc., Rochester, NY, USA). The diagnostic transducer was driven by a pulser-receiver system (Panametrics, Waltham, MA, USA), connected to a digitizer (Gage Applied Technologies, Inc., Lachine, QC, CAN) integrated into a personal computer (PC, Dell Inc., TX, USA). A custom coupling cone was designed and 3D-printed to fix the lens to the transducer and hold the coupling water.

Previous to the BBBO procedure, an acoustic calibration was carried out to experimentally evaluate the focal gain of the lens without the skull. Secondly, for the BBBO procedure, $1 \mu \mathrm{l} / \mathrm{g}$ Definity microbubbles were injected intravenously and 1.68-MHz FUS (400 kPa-PNP, PRF: $5 \mathrm{~Hz}$, pulse length: $1 \mathrm{~ms}$ ) was applied for 2 minutes [2], [18]. Then, $0.2 \mathrm{ml}$ of gadolinium tracer was injected intraperitoneally and posttreatment in vivo BBBO was assessed using T1-weighted MRI. Mice were transferred to the MRI scanner, anesthetized with $1-2 \%$ isoflurane, placed in a $3 \mathrm{~cm}$ birdcage coil and scanned with a small-animal 9.4T MRI system (Bruker, Billerica, MA, USA). A contrast-enhanced T1-weighted 2D FLASH scan (TR/TE: $230 / 3.3 \mathrm{~ms}$, flip angle: $70^{\circ}$, number of excitations: 18, in-plane resolution: $85 \mu \mathrm{m} \times 85 \mu \mathrm{m}$, slice thickness: 500 $\mu \mathrm{m}$, receiver bandwidth: $50 \mathrm{kHz}$ ) was acquired $\approx 30-45 \mathrm{~min}$ after FUS exposure, along both axial and coronal planes.

\section{Results}

First, the simulated axial pressure-field distribution with the mouse head [see Fig. 1(a,c)], normalized to the maximum
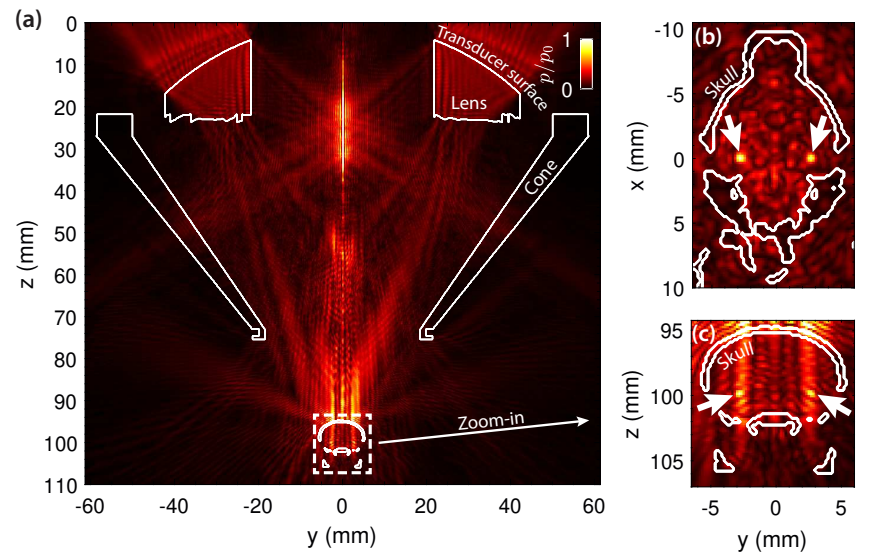

Fig. 1. Complete simulation of the pressure-field distribution. (a) Axial plane at $x=0 \mathrm{~mm}$. The contours of the lens, cone and skull are shown in continuous-white line. (b,c) Transversal plane at $z=100 \mathrm{~mm}$ and axial plane at $x=0 \mathrm{~mm}$ (zoom-in of (a)), respectively, showing the two focused spots by white arrows. 
pressure value at the focal region inside the brain $p_{0}$, and the simulated transversal plane [see Fig. 1(b)] show two distinct circular spots.

Then, T1-MRI shows gadolinium extravasation at two symmetric focal spots in the axial and coronal planes [see Fig. 2(a,b), respectively]. We observed a bilateral BBBO in all mice, with the highest contrast enhancement spots separated by $3.0 \pm 0.7 \mathrm{~mm}$ ( $n=5$ mice) compared to $5.3 \mathrm{~mm}$ in simulation. The discrepancy was likely due to the differences between the skull scanned with $\mu \mathrm{CT}$ for manufacturing the lens and the skulls of the treated mice.

Instead of generating ellipsoidal spots, as usual, we observe the generation of quasi-spherical spots, whose size is of the order of half the wavelength, as shown in both simulation [see Fig. 1(b,c)] and experiment [see Fig. 2(a,b)]. This effect emerges because the hologram encodes the direct timereversed field as well as the multiple reflections and reverberation generated by the skull cavity. In this way, the effective angular spectrum of the forward propagated field is increased as compared with a single FUS: the holographic system acts as an equivalent source with much higher aperture. The depth-offield of the focal spot for the simulated complete trans-cranial case is $\approx 0.5 \mathrm{~mm}$ (the wavelength in brain tissue is $0.95 \mathrm{~mm}$ ) compared to the $15 \mathrm{~mm}$ for the simulated calibration case [see Fig. 2(c)]. Furthermore, the FWHM in the transversal direction is $\approx 0.6 \mathrm{~mm}$ for the simulated complete trans-cranial case compared to $\approx 1 \mathrm{~mm}$ for the simulated calibration case [see Fig. 2(d)].

Note that at the beginning of the procedure, the small fnumber of the transducer $(N=0.73)$ made difficult the imaging of the lambda suture for the in-vivo setup. The lenses
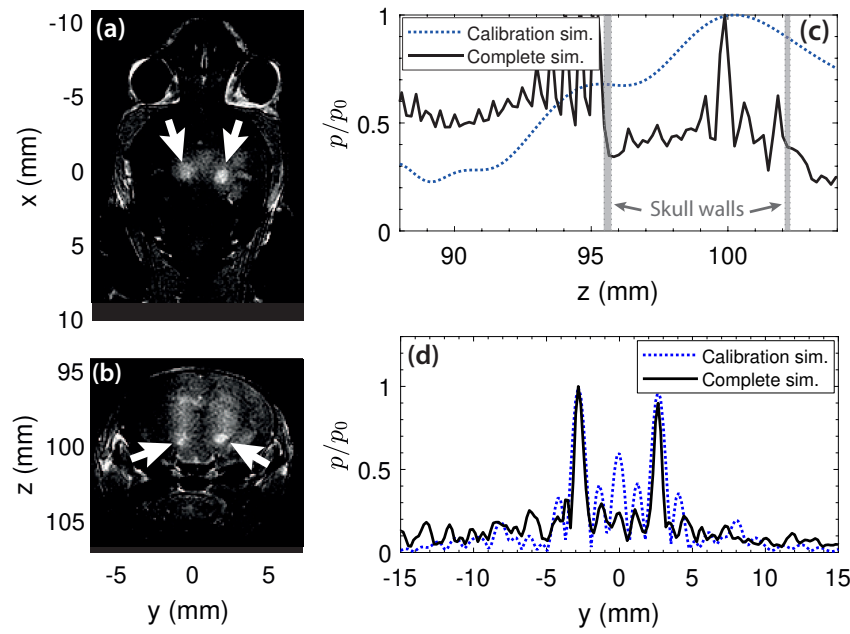

Fig. 2. Blood-brain barrier opening regions. (a,b) Experimental transversal and axial T1-MRI planes, respectively, showing the two areas of BBBO, in light color, by white arrows. (c) Simulated axial cross-section of the calibration case (dotted-blue line) and the complete simulation trans-cranial case (continuous-black line) at $y=2.6 \mathrm{~mm}$ and $x=0 \mathrm{~mm}$. (d) Simulated transversal cross-section of the calibration case (dotted-blue line) and the complete simulation trans-cranial case (continuous-black line) at $z=100$ $\mathrm{mm}$ and $x=0 \mathrm{~mm}$. developed in this work show how the focal distance can easily be adjusted, e.g., working at $F=100 \mathrm{~mm}(N=1.19)$ instead of the natural radius or curvature $F=61.65 \mathrm{~mm}$.

\section{CONCLUSION}

In this work, we demonstrate the first in-vivo multi-focal BBBO in mice using a 3D-printed holographic acoustic lens. The lens, which was effectively designed with a simulation using the $\mu \mathrm{CT}$-scan of a murine skull, showed the ability of simultaneous focusing at different brain regions of five mice, allowing thus a cost- and time-effective procedure with a onetime sonication and injection of microbubbles.

The high-pixel density lens allows the control of 131784 virtual source elements, an increase by about two orders of magnitude compared to state-of-the-art phased-array therapeutic systems (up to 2048), providing an excellent control of the acoustic field. Moreover, they are relatively low-cost compared to phased-array systems.

Holographic acoustic lenses allow a decrease in the number of sonications and microbubbles injections as they acoustically reconstruct the geometry of CNS structures. Moreover, they present an excellent quality focusing, i.e., spherical spots, since the lens is able to encode wave-field information from multiple reflections produced at the cranial cavity and, becoming in this way a high-localized technique for BBBO for the treatment of brain diseases.

This work shows the feasibility, capability and efficiency of holographic acoustic lenses for the highly-localized and multifocal BBBO. Furthermore, the benefits of this approach can be widely useful in other applications such as neuromodulation, chemotherapy or trans-cranial imaging, defining new paths for the complex requirements in the biomedical field.

\section{ACKNOWLEDGMENTS}

This research has been supported by the Spanish Ministry of Science, Innovation and Universities through grants "Juan de la Cierva - Incorporación” (IJC2018-037897-I) and PID2019111436RB-C22, by the Agència Valenciana de la Innovació through grant INNVAL10/19/016, by Generalitat Valenciana through grants No. ACIF/2017/045 and BEFPI/2019/075, and by the National Institutes of Health through grants 5R01EB009041 and 5R01AG038961. Action co-financed by the European Union through the Programa Operativo del Fondo Europeo de Desarrollo Regional (FEDER) of the Comunitat Valenciana 2014-2020 (IDIFEDER/2018/022).

\section{REFERENCES}

[1] J. Choi, M. Pernot, T. Brown, S. Small, and E. Konofagou, "Spatiotemporal analysis of molecular delivery through the blood-brain barrier using focused ultrasound," Physics in Medicine \& Biology, vol. 52, no. 18, p. 5509, 2007.

[2] Y.-S. Tung, F. Vlachos, J. A. Feshitan, M. A. Borden, and E. E. Konofagou, "The mechanism of interaction between focused ultrasound and microbubbles in blood-brain barrier opening in mice," The Journal of the Acoustical Society of America, vol. 130, no. 5, pp. 3059-3067, 2011. 
[3] A. N. Pouliopoulos, S.-Y. Wu, M. T. Burgess, M. E. Karakatsani, H. A. Kamimura, and E. E. Konofagou, "A clinical system for noninvasive blood-brain barrier opening using a neuronavigation-guided single-element focused ultrasound transducer," Ultrasound in medicine \& biology, vol. 46, no. 1, pp. 73-89, 2020.

[4] N. Lipsman, Y. Meng, A. J. Bethune, Y. Huang, B. Lam, M. Masellis, N. Herrmann, C. Heyn, I. Aubert, A. Boutet et al., "Blood-brain barrier opening in alzheimer's disease using mr-guided focused ultrasound," Nature communications, vol. 9, no. 1, pp. 1-8, 2018.

[5] R. Chopra, L. Curiel, R. Staruch, L. Morrison, and K. Hynynen, "An mricompatible system for focused ultrasound experiments in small animal models," Medical physics, vol. 36, no. 5, pp. 1867-1874, 2009.

[6] M. E. Poorman, V. L. Chaplin, K. Wilkens, M. D. Dockery, T. D. Giorgio, W. A. Grissom, and C. F. Caskey, "Open-source, smallanimal magnetic resonance-guided focused ultrasound system," Journal of therapeutic ultrasound, vol. 4, no. 1, p. 22, 2016.

[7] K. Melde, A. G. Mark, T. Qiu, and P. Fischer, "Holograms for acoustics," Nature, vol. 537, no. 7621, p. 518, 2016.

[8] S. Jiménez-Gambín, N. Jiménez, J. M. Benlloch, and F. Camarena, "Holograms to focus arbitrary ultrasonic fields through the skull," Physical Review Applied, vol. 12, no. 1, p. 014016, 2019.

[9] G. Maimbourg, A. Houdouin, T. Deffieux, M. Tanter and J.F. Aubry, "3D-printed adaptive acoustic lens as a disruptive technology for transcranial ultrasound therapy using single-element transducers," Physics in Medicine \& Biology, vol. 63, no. 2, p. 025026, 2018.

[10] S. Jiménez-Gambín, N. Jiménez, J. María. Benlloch, and F. Camarena, "Generating bessel beams with broad depth-of-field by using phase-only acoustic holograms," Scientific reports, vol. 9, no. 1, pp. 1-13, 2019.

[11] B. E. Treeby and B. Cox, "Modeling power law absorption and dispersion for acoustic propagation using the fractional laplacian," The Journal of the Acoustical Society of America, vol. 127, no. 5, pp. 2741-2748, 2010.

[12] N. Jiménez, F. Camarena, J. Redondo, V. Sánchez-Morcillo, Y. Hou, and E. E. Konofagou, "Time-domain simulation of ultrasound propagation in a tissue-like medium based on the resolution of the nonlinear acoustic constitutive relations," Acta Acustica united with Acustica, vol. 102, no. 5, pp. 876-892, 2016.

[13] U. Schneider, E. Pedroni, and A. Lomax, "The calibration of ct hounsfield units for radiotherapy treatment planning," Physics in Medicine \& Biology, vol. 41, no. 1, p. 111, 1996.

[14] T. D. Mast, "Empirical relationships between acoustic parameters in human soft tissues," Acoustics Research Letters Online, vol. 1, no. 2, pp. 37-42, 2000.

[15] J.-F. Aubry, M. Tanter, M. Pernot, J.-L. Thomas, and M. Fink, "Experimental demonstration of noninvasive transskull adaptive focusing based on prior computed tomography scans," The Journal of the Acoustical Society of America, vol. 113, no. 1, pp. 84-93, 2003.

[16] R. S. Cobbold, Foundations of biomedical ultrasound. Oxford University Press, 2006.

[17] P. A. Yushkevich, J. Piven, H. C. Hazlett, R. G. Smith, S. Ho, J. C. Gee, and G. Gerig, "User-guided 3d active contour segmentation of anatomical structures: significantly improved efficiency and reliability," Neuroimage, vol. 31, no. 3, pp. 1116-1128, 2006.

[18] K. Hynynen, N. McDannold, N. A. Sheikov, F. A. Jolesz, and N. Vykhodtseva, "Local and reversible blood-brain barrier disruption by noninvasive focused ultrasound at frequencies suitable for trans-skull sonications," Neuroimage, vol. 24, no. 1, pp. 12-20, 2005. 